\title{
ASSESSMENT OF PHARYNGEAL WIDTH AND LENGTH, POSITION OF HYOID BONE, IT'S SIZE AND INCLINATION OF SOFT PALATE IN DIFFERENT SKELETAL MALOCCLUSIONS IN UTTRAKHAND POPULATION
}

\author{
${ }^{1}$ Dr. Uzma Nazir, ${ }^{2}$ Dr. Rajarshi Basu, ${ }^{3}$ Dr. Dr.Pranshu, ${ }^{4}$ Dr. Ruchi Agrawal. \\ ${ }^{1}$ Orthodontist and Sleep Medicine Specialist at Siestabraces by city Dental clinic, Bohri kadal. Srinagar, J.K \\ ${ }^{2}$ Senior Lecturer, Department of Oral \& Maxillofacial Surgery, Awadh Dental College and Hospital, Jamshedpur, Jharkhand. \\ ${ }^{3}$ PG Resident, Department of Conservative Dentistry \& endodontics,Inderprastha Dental College \& Hospital, Ghaziabad, U.P. \\ ${ }^{4}$ Senior Lecturer, Department of Public Health Dentistry, New Horizon Dental College \& Research Institute, Bilaspur (C.G.)
}

\section{DOI: http://dx.doi.org/10.47937/SJCMDS.2021.1101}

\begin{abstract}
:
\section{Background:}

Uvulo-glosso-pharyngeal dimensions and size and position of associated structures are integral part of Orthodontic treatment planning. Since decades various authors have assessed airway dimensions by different methods and implied the results in diagnosis and treatment planning.
\end{abstract}

\section{Aims \& Objectives:}

The aim of this study was to investigate the uvulo-pharyngeal dimensions and position of hyoid bone in subjects with different anteroposterior jaw relationships.

\section{Method:}

Cephalometric radiographs of 30 subjects (age 15-25 years of Uttrakhand ethnic origin) were divided into three Groups accorging to ANB and Wits appraisal. Group 1, Skeletal Class I (ANB 1-4 and Wits Appraisal -1 to 0) Group 2, Skeletal Class II (ANB >4 and Wits Appraisal >1) \& Group 3, Skeletal Class III (ANB $<1$ and Wits Appraisal $<-1.5$ ). Statistical analysis was undertaken using one-way ANOVA to evaluate the mean and standard deviation in the three Groups and Post hoc Tukey test was done to compare the variables between the three Groups.

\section{Results:}

On an average, upper airway width was decreased in Group 2 and increased in Group $3(\mathrm{p}=0.028)$. Lower airway was increased in Group $3(\mathrm{p}=0.005)$. Velopharyngeal airway was decreased in Group 2 and increased in Group $3(\mathrm{p}=0.042)$.Thickness of soft palate was increased in Group $3(\mathrm{p}=0.0 .13)$. Hyoid bone was positioned poaterioinferiorly in Group 2 and Anterosuperiorly in Group 3(p=0.002).
In conclusion, uvulo-pharyngeal airway and position of hyoid bone are affected by anteroposterior skeletal patterns in Uttrakhand population despite of its multiethenicity.

\section{Keywords:}

Uvulo-pharyngeal dimensions, Hyoid bone, Skeletal pattern, OSA (Obstructive Sleep Apnoea).

\section{Introduction:}

Pharynx, a tube-shaped structure formed by muscles and membranes, is located behind nasal and oral cavities, and extends from the cranial base to the level of the sixth cervical vertebra. 1 The pharyngeal airway is the first component of the significant structures, which provides respiration - one of the vital functions of the human body. Because of the close anatomical, structural and functional relationship between the pharynx, soft palate, tongue, epiglottis, hyoid Bone and craniofacial \& dentofacial structures such as cranial base, maxilla and mandible, a mutual interaction in terms of growth and function between them is expected. ${ }^{2}$

Pharyngeal airway space size is determined primarily by relative growth and size of the soft tissues surrounding the dentofacial skeleton. Studies have shown that the pharyngeal airway space is reduced, than normal in individuals with short cranial base and in cases of retrognathia or micrognathia. Angle showed that Class II Division 1 malocclusion was associated with upper airway obstruction and mouth breathing. Mergen and Jacobs reported that nasopharyngeal depth was significantly larger in patient with normal occlusion than in Class II malocclusion. ${ }^{1}$

In addition, different anatomic features of the maxilla and mandible could change the position of the hyoid and soft palate and lead to decreased dimension of posterior airway space. Suprahyoid muscles and infrahyoid muscles and their effect on the the shape, growth, external and internal angles of mandible have been less 
considered. Hyoid bone as a link between suprahyoid and infrahyoid muscles has a significant role in orientation and even in function of these muscles which should not be disregarded.3 Significant difference in position of hyoid bone was found between Class I, Class II and Class III malocclusions.4 It is further documented that soft palate length, thickness, and inclination is associated with various skeletal malocclusions.5 Since Orthodontists deal with various kinds of malocclusions, including severe skeletal Class II and III deformities and the standard procedures for correction of these discrepancies, it has therefore become mandatory to consider Pharyngeal Airway in Diagnosis and Treatment Planning to eliminate the disturbances in respiration, as disturbed breathing function could lead to life threatening situations such as obstructive sleep apnea (OSA), which is characterized by recurrent episodes of upper airway obstruction during sleep resulting in reduced oxygen saturation and systemic disorders such as Cor pulmonale, thus associated with increased morbidity and mortality. ${ }^{6}$

As the relationship between the Pharyngeal Airway and changes in facial morphology has been extensively debated in the literature and still remained controversial, therefore in view of the need to uncover new evidences to contribute to and assist in addressing this complex issue, this study was carried out by tracing certain reference points, lines and angles on digitalized lateral cephalograms to evaluate the variations in pharyngeal airway spaces, position of hyoid bone and size and inclination of soft palate in skeletal Class I, Class II and Class III malocclusions in Uttrakhand population and to derive clinical implications of study as applicable to Orthodontic treatment planning.

\section{Materials \& Methods:}

The present cephalometric study was done on untreated Orthodontic patients Uttrakhand ethnicity selected from the subjects who visited to the OPD at Department of Orthodontics and Dentofacial Orthopaedics, Seema Dental College and Hospital, Rishikesh.

The study comprised of 30 subjects aged between 15-25 years, breathing comfortably through nose. All digital Cephalometric radiographs were taken in NHP and patients were asked to swallow while shooting for cephalogram. All Cephalograms were taken using Kodak 8000C Panoramic \& Cephalometric unit at Tube voltage of $60-90 \mathrm{kVp}$, Digital censor CCD with 1360X1840 Pixels and magnification of 1:1\%.

Lateral cephalometric radiographs were traced on acetate paper and 26 hard tissue and 14 soft tissue reference points were registered yielding 4 angular and 17 linear measurements. Sample was divided into three groups with 10 subjects in each group.

- Group 1, Skeletal Class I (ANB 1-4 and Wits Appraisal -1 to 0$)$
- Group 2, Skeletal Class II (ANB >4 and Wits Appraisal $>1)$

- Group 3, Skeletal Class III (ANB $<1$ and Wits Appraisal < $-1.5)$

To minimise the error, all the cephalograms were manually traced and analysed by a single observer. Three radiographs from each group were randomly selected and revaluated by the same observer to reduce Intra-observer error.

\section{Inclusion Criteria:}

1. Age should be in the range of 15 to 25 years with mean Age being 20 years.

2. Native Uttrakhand population based upon history and domicile obtained from the parents in the form of a questionnaire format.

3. Skeletal Class I, Class II and Class III malocclusion. - Evaluated by cephalometric analysis.

4. Subjects able to breath comfortably through nose.

Subjects with history of tonsilitis and pharyngitis, major trauma to craniofacial region, orthognathic surgery,mouth breathing and previous orthodontics treatment were excluded.

Cephalometric landmarks, reference lines, parameters to assess pharyngeal airway, soft palate and position of hyoid bone are given in Diagram 1,2,3,4 \& 5 respectively.

LANDMARKS USED IN STUDY

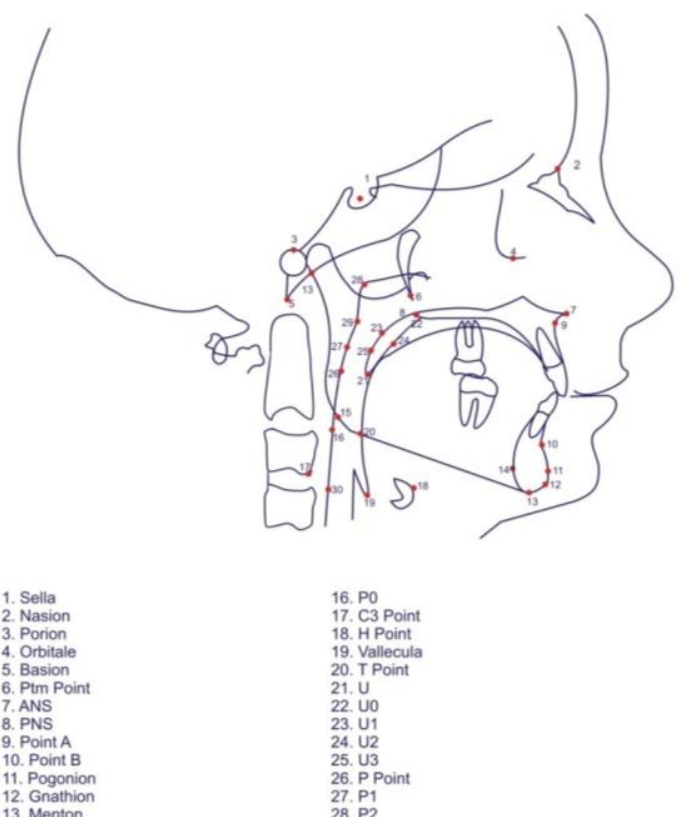


REFERENCE PLANE USED IN STUDY

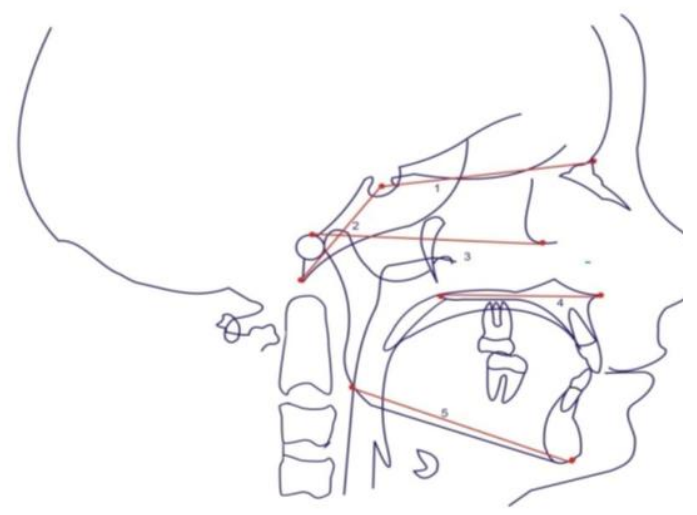

1. Sella Nasion Plane 3. Frankfort Horizontal Plane 4. Palatal Plane
5. Mandibular Plane

PARAMETERS USED FOR ASSESSMENT OF PHARYNGEAL AIRWAY

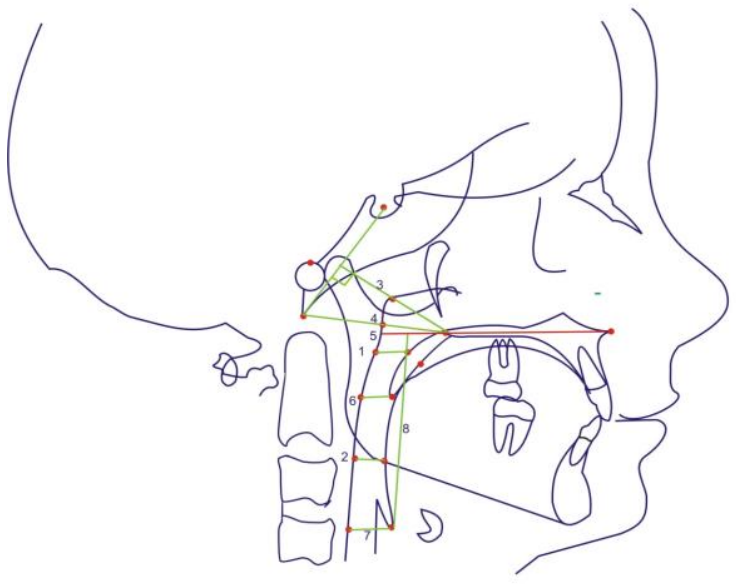

1. Upper Airway

2. Lower Airway

4. Lower Airway Width

5. Nasopharyngeal Airway Depth 6. Oropharyngeal Airway Depth

7.. Vertical Pharyngeal Length
8. Veal

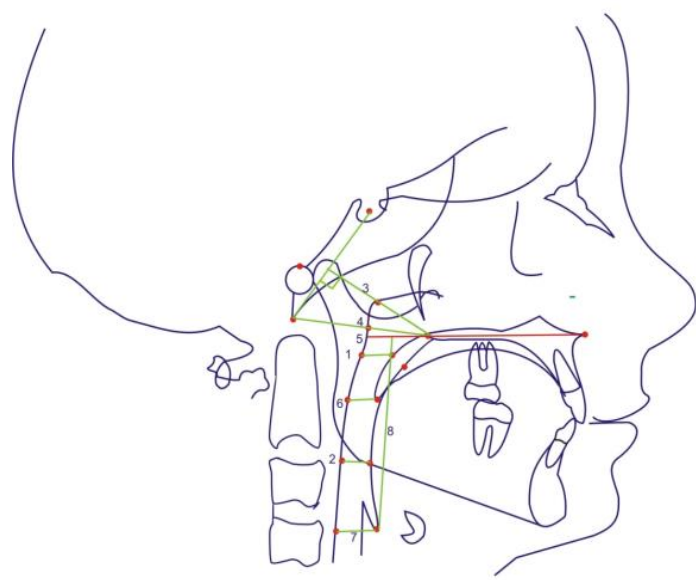

Upper Airway

3. Upper Airway Width

4. Lower Airway Width

5. Nasopharyngeal Airway Depth 6. Oropharyngeal Airway Depth 7..Velopharyngeal Airway Depth 8. Vertical Pharyngeal Length

PARAMETERS USED FOR ASSESSMENT OF SOFT PALATE

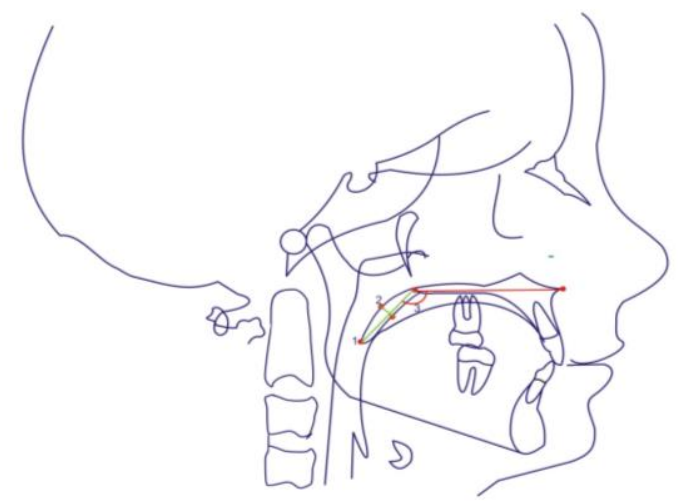

1. Length of Soft Palate 2. Thickness of Soft Palate
3. Inclination of Soft Palate 


\section{Statistical Analysis:}

Descriptive statistics including mean and standard deviation for each Group were computed using one-way ANOVA to determine whether significant differences exist between the three groups. The subgroup Analysis between Class I and Class II, Class I and Class III, Class II and Class III was done using Post hoc Tukey test.

\section{Results:}

Dahlberg and Houston analysis showed that systematic error was not significant $(\mathrm{P}=0.321)$; therefore, the measurements were highly reproducible. The results of the statistical analysis with mean, Standard deviation, and p values are shown in the (Table 1.ad \& Table 2.a-d) \& (Graph a).

Table 1.a Parameters to assess anteroposterior skeletal relationship of the subjects in

\section{Group 1, Group 2 and Group 3 by One-way ANOVA}

\begin{tabular}{|c|c|c|c|c|c|c|c|}
\hline Parameters & Groups & $\mathbf{N}$ & Mean & $\begin{array}{c}\text { Std. } \\
\text { Deviation }\end{array}$ & $\begin{array}{c}\text { Statistics/ } \\
\text { mean } \\
\text { squares }\end{array}$ & $\begin{array}{l}\text { df2(welch) } \\
\text { / F(Anova) }\end{array}$ & $\begin{array}{l}\text { Anova } \\
\text { P Value }\end{array}$ \\
\hline$\overline{\text { ANB }}$ & Class I & 10 & 2.2 & 1.2517 & \multirow{4}{*}{155.465} & \multirow{4}{*}{16.788} & \multirow{4}{*}{$<0.001$} \\
\hline & Class II & 10 & 7 & 1.8257 & & & \\
\hline & Class III & 10 & -3.45 & 0.896 & & & \\
\hline & Total & 30 & 1.917 & 4.5431 & & & \\
\hline \multirow[t]{4}{*}{ WA } & Class I & 10 & 0.85 & 0.8182 & \multirow{4}{*}{262.8} & \multirow{4}{*}{163.965} & \multirow{4}{*}{$<0.001$} \\
\hline & Class II & 10 & 5.05 & 1.3427 & & & \\
\hline & Class III & 10 & -5.15 & 1.5284 & & & \\
\hline & Total & 30 & 0.25 & 4.429 & & & \\
\hline
\end{tabular}

Table 1.b Parameters to assess Pharyngeal airway of the subjects in group 1, group 2 and group 3 by One-way ANOVA

\begin{tabular}{|c|c|c|c|c|c|c|c|}
\hline Parameters & Groups & $\mathbf{N}$ & Mean & $\begin{array}{c}\text { Std. } \\
\text { Deviation }\end{array}$ & $\begin{array}{c}\text { Statistics/ } \\
\text { mean } \\
\text { squares }\end{array}$ & $\begin{array}{l}\text { df2(welch) } \\
\text { / F(Anova) }\end{array}$ & $\begin{array}{l}\text { Anova } \\
\text { P Value }\end{array}$ \\
\hline \multicolumn{8}{|c|}{ Linear Measurements } \\
\hline \multirow{4}{*}{ UA } & Class I & 10 & 11.75 & 2.486 & \multirow{4}{*}{7.234} & \multirow{4}{*}{13.045} & \multirow{4}{*}{0.032} \\
\hline & Class II & 10 & 11 & 2.041 & & & \\
\hline & Class III & 10 & 13.3 & 0.483 & & & \\
\hline & Total & 30 & 12.02 & 2.057 & & & \\
\hline
\end{tabular}


Uzma, et al.: Assessment of pharyngeal width and length....

\begin{tabular}{|c|c|c|c|c|c|c|c|}
\hline \multirow{4}{*}{ LA } & Class I & 10 & 7.65 & 1.886 & \multirow{4}{*}{21.1} & \multirow{4}{*}{6.101} & \multirow{4}{*}{0.007} \\
\hline & Class II & 10 & 9.25 & 2.475 & & & \\
\hline & Class III & 10 & 10.55 & 0.832 & & & \\
\hline & Total & 30 & 9.15 & 2.162 & & & \\
\hline \multirow{4}{*}{ UAW } & Class I & 10 & 15.55 & 3.166 & \multirow{4}{*}{0.758} & \multirow{4}{*}{0.143} & \multirow{4}{*}{0.867} \\
\hline & Class II & 10 & 15.85 & 1.959 & & & \\
\hline & Class III & 10 & 15.3 & 1.418 & & & \\
\hline & Total & 30 & 15.57 & 2.231 & & & \\
\hline \multirow{4}{*}{ LAW } & Class I & 10 & 20.5 & 2.369 & \multirow{4}{*}{5.658} & \multirow{4}{*}{0.781} & \multirow{4}{*}{0.468} \\
\hline & Class II & 10 & 20.6 & 2.923 & & & \\
\hline & Class III & 10 & 19.25 & 2.751 & & & \\
\hline & Total & 30 & 20.12 & 2.671 & & & \\
\hline \multirow{4}{*}{ NAD } & Class I & 10 & 20.2 & 2.474 & \multirow{4}{*}{1.608} & \multirow{4}{*}{0.277} & \multirow{4}{*}{0.76} \\
\hline & Class II & 10 & 20.55 & 2.179 & & & \\
\hline & Class III & 10 & 19.75 & 2.563 & & & \\
\hline & Total & 30 & 20.17 & 2.35 & & & \\
\hline \multirow{4}{*}{ OAD } & Class I & 10 & 8.1 & 2.951 & \multirow{4}{*}{12.233} & \multirow{4}{*}{1.915} & \multirow{4}{*}{0.167} \\
\hline & Class II & 10 & 9 & 2.357 & & & \\
\hline & Class III & 10 & 10.3 & 2.214 & & & \\
\hline & Total & 30 & 9.13 & 2.606 & & & \\
\hline \multirow{4}{*}{ VAD } & Class I & 10 & 11.8 & 3.889 & \multirow{4}{*}{4.64} & \multirow{4}{*}{13.982} & \multirow{4}{*}{0.007} \\
\hline & Class II & 10 & 16.6 & 3.836 & & & \\
\hline & Class III & 10 & 12.9 & 1.197 & & & \\
\hline & Total & 30 & 13.77 & 3.75 & & & \\
\hline \multicolumn{8}{|c|}{ Angular Measurements } \\
\hline \multirow{4}{*}{ VPL } & Class I & 10 & 48.05 & 3.444 & \multirow{4}{*}{9.158} & \multirow{4}{*}{0.297} & \multirow{4}{*}{0.746} \\
\hline & Class II & 10 & 46.2 & 6.889 & & & \\
\hline & Class III & 10 & 46.7 & 5.774 & & & \\
\hline & Total & 30 & 46.98 & 5.421 & & & \\
\hline
\end{tabular}


Uzma, et al.: Assessment of pharyngeal width and length....

Table 1.c Parameters to assess position of Hyoid bone in the subjects in group 1, group 2 \& group 3 by One-way ANOVA

\begin{tabular}{|c|c|c|c|c|c|c|c|}
\hline Parameters & Groups & $\mathbf{N}$ & Mean & $\begin{array}{c}\text { Std. } \\
\text { Deviation }\end{array}$ & $\begin{array}{c}\text { Statistics/ } \\
\text { mean } \\
\text { squares }\end{array}$ & $\begin{array}{l}\text { df2(welch) } \\
\text { / F(Anova) }\end{array}$ & $\begin{array}{l}\text { Anova } \\
\text { P Value }\end{array}$ \\
\hline \multicolumn{8}{|c|}{ Linear Measurements } \\
\hline \multirow{4}{*}{ C3/RGs - H } & Class I & 10 & 0 & 4 & \multirow{4}{*}{19.308} & \multirow{4}{*}{0.57} & \multirow{4}{*}{0.572} \\
\hline & Class II & 10 & 2.15 & 6.3336 & & & \\
\hline & Class III & 10 & -0.45 & 6.7472 & & & \\
\hline & Total & 30 & 0.567 & 5.7336 & & & \\
\hline \multirow{4}{*}{ H-RGs } & Class I & 10 & 30.65 & 5.972 & \multirow{4}{*}{1.908} & \multirow{4}{*}{0.061} & \multirow{4}{*}{0.941} \\
\hline & Class II & 10 & 30.4 & 3.526 & & & \\
\hline & Class III & 10 & 29.8 & 6.746 & & & \\
\hline & Total & 30 & 30.28 & 5.402 & & & \\
\hline \multirow{4}{*}{ MP-H } & Class I & 10 & -6.6 & 3.1693 & \multirow{4}{*}{13.733} & \multirow{4}{*}{0.561} & \multirow{4}{*}{0.577} \\
\hline & Class II & 10 & -8.8 & 5.1381 & & & \\
\hline & Class III & 10 & -8.4 & 6.0773 & & & \\
\hline & Total & 30 & -7.933 & 4.8703 & & & \\
\hline \multirow{4}{*}{$\mathrm{C} 3-\mathrm{H}$} & Class I & 10 & 30.4 & 3.6878 & \multirow{4}{*}{10.033} & \multirow{4}{*}{0.504} & \multirow{4}{*}{0.61} \\
\hline & Class II & 10 & 30 & 5.1208 & & & \\
\hline & Class III & 10 & 31.9 & 4.4585 & & & \\
\hline & Total & 30 & 30.767 & 4.384 & & & \\
\hline \multirow{4}{*}{ H/N-FHP } & Class I & 10 & 43.05 & 6.379 & \multirow{4}{*}{174.925} & \multirow{4}{*}{4.231} & \multirow{4}{*}{0.025} \\
\hline & Class II & 10 & 48.1 & 7.989 & & & \\
\hline & Class III & 10 & 39.8 & 4.417 & & & \\
\hline & Total & 30 & 43.65 & 7.11 & & & \\
\hline \multicolumn{8}{|c|}{ Angular Measurements } \\
\hline \multirow{4}{*}{$\mathrm{NSH}$} & Class I & 10 & 87.45 & 5.014 & \multirow{4}{*}{71.858} & \multirow{4}{*}{4.125} & \multirow{4}{*}{0.027} \\
\hline & Class II & 10 & 90.8 & 4.104 & & & \\
\hline & Class III & 10 & 85.5 & 3.206 & & & \\
\hline & Total & 30 & 87.92 & 4.602 & & & \\
\hline
\end{tabular}




\begin{tabular}{|c|c|c|c|c|c|c|c|}
\hline \multirow{4}{*}{$\begin{array}{l}\text { MPH } \\
\text { Angle }\end{array}$} & Class I & 10 & 14.9 & 5.071 & \multirow{4}{*}{2.533} & \multirow{4}{*}{0.048} & \multirow{4}{*}{0.953} \\
\hline & Class II & 10 & 15.9 & 6.154 & & & \\
\hline & Class III & 10 & 15.5 & 9.744 & & & \\
\hline & Total & 30 & 15.43 & 7.027 & & & \\
\hline \multirow{4}{*}{ FH-H } & Class I & 10 & 67.3 & 4.00832 & \multirow{4}{*}{6.175} & \multirow{4}{*}{0.233} & \multirow{4}{*}{0.794} \\
\hline & Class II & 10 & 65.85 & 6.12849 & & & \\
\hline & Class III & 10 & 67.1 & 5.10338 & & & \\
\hline & Total & 30 & 66.75 & 5.01506 & & & \\
\hline
\end{tabular}

Table 1.d Parameters to assess the size and inclination of soft palate in the subjects in group 1, group 2 and group 3 by One-way ANOVA.

\begin{tabular}{|c|c|c|c|c|c|c|c|}
\hline Parameters & Groups & $\mathbf{N}$ & Mean & $\begin{array}{c}\text { Std. } \\
\text { Deviation }\end{array}$ & $\begin{array}{c}\text { Statistics/ } \\
\text { mean } \\
\text { squares }\end{array}$ & $\begin{array}{l}\text { df2(welch) } \\
\text { / F(Anova) }\end{array}$ & $\begin{array}{l}\text { Anova } \\
\text { P Value }\end{array}$ \\
\hline \multicolumn{8}{|c|}{ Linear Measurements } \\
\hline \multirow{4}{*}{ LSP } & Class I & 10 & 27.2 & 3.393 & \multirow{4}{*}{0.951} & \multirow{4}{*}{15.745} & \multirow{4}{*}{0.28} \\
\hline & Class II & 10 & 26.2 & 1.619 & & & \\
\hline & Class III & 10 & 25.05 & 3.444 & & & \\
\hline & Total & 30 & 26.15 & 2.977 & & & \\
\hline \multirow{4}{*}{ TSP } & Class I & 10 & 7.4 & 0.568 & \multirow{4}{*}{6.358} & \multirow{4}{*}{4.972} & \multirow{4}{*}{0.015} \\
\hline & Class II & 10 & 6.3 & 1.567 & & & \\
\hline & Class III & 10 & 7.85 & 1.029 & & & \\
\hline & Total & 30 & 7.18 & 1.276 & & & \\
\hline \multicolumn{8}{|c|}{ Angular Measurements } \\
\hline \multirow{4}{*}{ ISP } & Class I & 10 & 124.25 & 6.957 & \multirow{4}{*}{239.925} & \multirow{4}{*}{3.292} & \multirow{4}{*}{0.053} \\
\hline & Class II & 10 & 132.5 & 9.629 & & & \\
\hline & Class III & 10 & 123.8 & 8.804 & & & \\
\hline & Total & 30 & 126.85 & 9.187 & & & \\
\hline
\end{tabular}


Table 2.a Parameters to compare anteroposterior skeletal relationship in group 1, group 2 and group 3 by poat hoc tukey test.

\begin{tabular}{|c|c|c|c|c|c|}
\hline $\begin{array}{c}\text { Dependent } \\
\text { Variable }\end{array}$ & (I) Group & (J) Group & $\begin{array}{c}\text { Mean } \\
\text { Difference (I-J) }\end{array}$ & Std. Error & P Value \\
\hline \multirow{3}{*}{ ANB } & \multirow{3}{*}{ Class I } & Class II & $-4.8000^{*}$ & 0.6166 & $<0.001$ \\
\cline { 2 - 6 } & Class II & Class III & $5.6500^{*}$ & 0.6166 & $<0.001$ \\
\cline { 2 - 6 } & \multirow{2}{*}{ Class I } & Class III & $10.4500^{*}$ & 0.6166 & $<0.001$ \\
\cline { 2 - 6 } & Class II & $-4.2000^{*}$ & 0.5662 & $<0.001$ \\
\cline { 2 - 6 } & Class II & Class III & $6.0000^{*}$ & 0.5662 & $<0.001$ \\
\hline \multirow{2}{*}{ WA } & & & $10.2000^{*}$ & 0.5662 & $<0.001$ \\
\hline
\end{tabular}

Table 2.b Parameters to compare pharyngeal airway in group 1, group 2 and group 3 by post hoc tukey test.

\begin{tabular}{|c|c|c|c|c|c|}
\hline $\begin{array}{c}\text { Dependent } \\
\text { Variable }\end{array}$ & (I) Group & (J) Group & $\begin{array}{c}\text { Mean } \\
\text { Difference (I-J) }\end{array}$ & Std. Error & P Value \\
\hline \multicolumn{6}{|c|}{ Linear Measurements } \\
\hline \multirow{3}{*}{ UA } & \multirow[b]{2}{*}{ Class I } & Class II & 0.75 & 0.84 & 0.649 \\
\hline & & Class III & -1.55 & 0.84 & 0.174 \\
\hline & Class II & Class III & $-2.300 *$ & 0.84 & 0.028 \\
\hline \multirow{3}{*}{ LA } & \multirow[b]{2}{*}{ Class I } & Class II & -1.6 & 0.832 & 0.151 \\
\hline & & Class III & $-2.900 *$ & 0.832 & 0.005 \\
\hline & Class II & Class III & -1.3 & 0.832 & 0.279 \\
\hline \multirow{3}{*}{ UAW } & \multirow[b]{2}{*}{ Class I } & Class II & -0.3 & 1.029 & 0.954 \\
\hline & & Class III & 0.25 & 1.029 & 0.968 \\
\hline & Class II & Class III & 0.55 & 1.029 & 0.855 \\
\hline \multirow{3}{*}{ LAW } & \multirow[b]{2}{*}{ Class I } & Class II & -0.1 & 1.203 & 0.996 \\
\hline & & Class III & 1.25 & 1.203 & 0.559 \\
\hline & Class II & Class III & 1.35 & 1.203 & 0.509 \\
\hline \multirow{3}{*}{ NAD } & \multirow[b]{2}{*}{ Class I } & Class II & -0.35 & 1.078 & 0.944 \\
\hline & & Class III & 0.45 & 1.078 & 0.909 \\
\hline & Class II & Class III & 0.8 & 1.078 & 0.741 \\
\hline
\end{tabular}




\begin{tabular}{|c|c|c|c|c|c|}
\hline \multirow{3}{*}{ OAD } & \multirow[b]{2}{*}{ Class I } & Class II & -0.9 & 1.13 & 0.709 \\
\hline & & Class III & -2.2 & 1.13 & 0.145 \\
\hline & Class II & Class III & -1.3 & 1.13 & 0.493 \\
\hline \multirow{3}{*}{ VAD } & \multirow[b]{2}{*}{ Class I } & Class II & $-4.800 *$ & 1.444 & 0.007 \\
\hline & & Class III & -1.1 & 1.444 & 0.729 \\
\hline & Class II & Class III & $3.700 *$ & 1.444 & 0.042 \\
\hline \multicolumn{6}{|c|}{ Angular Measurements } \\
\hline \multirow{3}{*}{ VPL } & \multirow[b]{2}{*}{ Class I } & Class II & 1.85 & 2.485 & 0.74 \\
\hline & & Class III & 1.35 & 2.485 & 0.851 \\
\hline & Class II & Class III & -0.5 & 2.485 & 0.978 \\
\hline
\end{tabular}

Table 2.c Parameters to compare the position of hyoid bone in group 1, group 2 and group 3 by post hoc tukey test

\begin{tabular}{|c|c|c|c|c|c|}
\hline $\begin{array}{c}\text { Dependent } \\
\text { Variable }\end{array}$ & (I) Group & (J) Group & $\begin{array}{c}\text { Mean } \\
\text { Difference (I-J) }\end{array}$ & Std. Error & P Value \\
\hline \multicolumn{6}{|c|}{ Linear Measurements } \\
\hline \multirow{3}{*}{ C3/RGs - H } & \multirow[b]{2}{*}{ Class I } & Class II & -2.15 & 2.6031 & 0.69 \\
\hline & & Class III & 0.45 & 2.6031 & 0.984 \\
\hline & Class II & Class III & 2.6 & 2.6031 & 0.584 \\
\hline \multirow{3}{*}{ H-RGs } & \multirow[b]{2}{*}{ Class I } & Class II & 0.25 & 2.498 & 0.994 \\
\hline & & Class III & 0.85 & 2.498 & 0.938 \\
\hline & Class II & Class III & 0.6 & 2.498 & 0.969 \\
\hline \multirow{3}{*}{ MP-H } & \multirow[b]{2}{*}{ Class I } & Class II & 2.2 & 2.2118 & $<0.001$ \\
\hline & & Class III & 1.8 & 2.2118 & 0.586 \\
\hline & Class II & Class III & -0.4 & 2.2118 & 0.698 \\
\hline \multirow[t]{3}{*}{$\mathrm{C} 3-\mathrm{H}$} & \multirow[b]{2}{*}{ Class I } & Class II & 0.4 & 1.995 & 0.978 \\
\hline & & Class III & -1.5 & 1.995 & 0.735 \\
\hline & Class II & Class III & -1.9 & 1.995 & 0.613 \\
\hline
\end{tabular}


Uzma, et al.: Assessment of pharyngeal width and length....

\begin{tabular}{|c|c|c|c|c|c|}
\hline \multirow{3}{*}{ H/N-FHP } & \multirow[b]{2}{*}{ Class I } & Class II & -5.05 & 2.875 & 0.203 \\
\hline & & Class III & 3.25 & 2.875 & 0.504 \\
\hline & Class II & Class III & $8.300^{*}$ & 2.875 & 0.02 \\
\hline \multicolumn{6}{|c|}{ Angular Measurements } \\
\hline \multirow{3}{*}{ NSH } & \multirow[b]{2}{*}{ Class I } & Class II & -3.35 & 1.867 & 0.19 \\
\hline & & Class III & 1.95 & 1.867 & 0.556 \\
\hline & Class II & Class III & $5.300 *$ & 1.867 & 0.022 \\
\hline \multirow[b]{2}{*}{ MPH } & \multirow[b]{2}{*}{ Class I } & Class II & -1 & 3.251 & 0.949 \\
\hline & & Class III & -0.6 & 3.251 & 0.981 \\
\hline Angle & Class II & Class III & 0.4 & 3.251 & 0.992 \\
\hline \multirow{3}{*}{ FH-H } & \multirow[b]{2}{*}{ Class I } & Class II & 1.45 & 2.30462 & 0.805 \\
\hline & & Class III & 0.2 & 2.30462 & 0.996 \\
\hline & Class II & Class III & -1.25 & 2.30462 & 0.851 \\
\hline
\end{tabular}

Table 2.d Parameters to compare the size and inclination of soft palate in group 1, group 2 and group 3 by post hoc tukey test

\begin{tabular}{|c|c|c|c|c|c|}
\hline $\begin{array}{l}\text { Dependent } \\
\text { Variable }\end{array}$ & (I) Group & (J) Group & $\begin{array}{c}\text { Mean } \\
\text { Difference (I-J) }\end{array}$ & Std. Error & P Value \\
\hline \multicolumn{6}{|c|}{ Linear Measurements } \\
\hline \multirow{3}{*}{ LSP } & \multirow[b]{2}{*}{ Class I } & Class II & 1 & 1.316 & 0.73 \\
\hline & & Class III & 2.15 & 1.316 & 0.249 \\
\hline & Class II & Class III & 1.15 & 1.316 & 0.661 \\
\hline \multirow{3}{*}{ TSP } & \multirow[b]{2}{*}{ Class I } & Class II & 1.1 & 0.506 & 0.094 \\
\hline & & Class III & -0.45 & 0.506 & 0.651 \\
\hline & Class II & Class III & $-1.550^{*}$ & 0.506 & 0.013 \\
\hline \multicolumn{6}{|c|}{ Angular Measurements } \\
\hline \multirow{3}{*}{ ISP } & \multirow[b]{2}{*}{ Class I } & Class II & -8.25 & 3.818 & 0.097 \\
\hline & & Class III & 0.45 & 3.818 & 0.992 \\
\hline & Class II & Class III & 8.7 & 3.818 & 0.076 \\
\hline
\end{tabular}


Results statistically significant for Upper Airway between Group 2 \& Group 3 but no significance was found when Group 1 was compared to Group $2 \&$ Group 3. It was found to be increased in Group 3(13.3mm) and decreased in Group 2 $(11 \mathrm{~mm})(\mathrm{p}=0.028)$. However statistical significance of Lower Airway was found to be decreased in Group $1(7.76 \mathrm{~mm})$ and increased in Group $3(10.55 \mathrm{~mm})(\mathrm{p}=.005) \&$ no positive statistical significance existed when Group 2 was compared to Group $1 \&$ Group 3. Statistically significant values were found in Velopharyngeal Airway Depth between Group 1(11.8mm) and Group 2(16.6mm) and between Group 2(16.6mm) and Group 3(12.9mm) however no statistical significance was seen between Group 1 and Group 3. It was found to be decreased in Group 1 \& Group 3 and increased in Group $2(\mathrm{p}=.007)$ $(p=0.042)$ respectively. Thickness of soft palate was found to be decreased in Group $2(6.3 \mathrm{~mm})$ and increased in Group $3(7.85 \mathrm{~mm})(\mathrm{p}=.015)$, however no statistical significant values were found when Group 1 was compared to Group 2 and Group 3. The highly significant values were found in MP-H between Group 1(-6.6mm) and Group $2(-8.8 \mathrm{~mm})(\mathrm{p}=0.001)$ and it was found that hyoid bone was inferiorly positioned in relation to Mandibular plane in Group 2, however statistically no significance existed when Group 1 was compared to Group 2 and Group 3 and when Group 2 and Group 3 were compared. The values for H/N-FHP were found to be statistically significant between Group 2 and Group 3 with increase in Group 2(48.1mm) and decreased in Group $3(39.8 \mathrm{~mm})$ $(\mathrm{p}=0.02)$. NSH was found to be increased in Group 2 (90.8 degrees) and decreased in Group 3 (85.5degrees) $(\mathrm{p}=0.02)$. No statistical significance existed in $\mathrm{H} / \mathrm{N}-\mathrm{FH} \& \mathrm{NSH}$ values when Group 1 was compared to Group 2 and Group 3. No statistical significance was found in relation of UAW, LAW, NAD, OAD, VPL, LSP, ISP, C3/RGs-H, H-RGs, MP-H, C3-H, MPH and $\mathrm{FH}-\mathrm{H}$ to various sagittal skeletal malocclusions.

\section{Discussion:}

Uttrakhand, the 27th state of Republic of India is on an average 2,084 mts above sea level. Voluntary hyperventilation and breath holding at high altitudes has been documented. Voluntary hyperventilation and breath holding produce respiratory and cardiac changes. The great variation has been found in the pattern of breathing and respiratory indices in the population residing at high altitudes.7 Therefore Uttrakhand population was choosen for the study to evaluate whether any airway changes with the change in ANB in this very population is similar to those of documented earlier or any deviation exists.

Cephalogram is a standardized lateral radiograph of head and neck used to examine upper airway, craniofacial and soft tissue structures all togather. Lateral cephalometry is widely available, easily performed and much less expensive than CT Scanning and MRI with least radiation exposure to the subjects. Posterior airway space, as measured by lateral cephalograms, was highly correlated with measurements using 3D - CT scan, with $92 \%$ accuracy in predictability. ${ }^{8}$
ANB \& Wits Appraisal as the indicators of sagittal divergence were used to divide the sample into three Groups. ANB is the most commonly used and most accurate and reliable measurement for appraising anteroposterior disharmony of the jaws as per studies conducted by Oktay \& Ishikawa et al. The reference points can easily be located on a cephalogram \& repeatedly reproduced.9,10 Wits Appraisal was used as adjunctive measurement of anteroposterior jaw relations in order to obviate the shortcomings of ANB such as unstable antero posterior position of nasion and rotational growth of the jaws as per studies conducted by Jacobson, Hussel and Nanda.11,12 Since Wits appraisal includes functional occlusal plane as reference line which was traditionally being used by all phases of dentistry including Angle and it intimately related to Point A and point B and to the masticatory forces, thus holding a firm importance in sampling.

The nasopharyngeal dimensions continue to grow rapidly until 13 years of age and then slowly until adulthood. In this study the age range was 15-25 years with the mean age of 20 years to ensure that the oropharyngeal structures had reached adult size. ${ }^{5}$

As it has been suggested that the head posture influence the dimensions of pharyngeal airway, so all the cephalometric radiographs of the subjects were recorded with the head in NHP (Natural Head Position). ${ }^{13}$

A normal nasal airway is dependent on sufficient anatomical dimensions of airway. Experimental studies carried out by Harvold $\&$ associates using primates showed varied dentofacial forms \& malocclusions, resulting after establishment of mouth breathing. On the other hand it has been mentioned in the literature that malocclusion type does not influence pharyngeal width (Watson et al., 1968; de Freitas et al., 2006; Alves et al., 2008).14 Since the correlation of facial morphology to that of airway dimensions is still a controversy therefore the present study was conducted to find whether any changes are evident in pharyngeal airway, position of hyoid bone and dimensions \& inclination of soft palate in relation to various anteroposterior skeletal malocclusions in Uttrakhand population.

The present study showed that the upper pharyngeal airway was found to be decreased in Class II subjects \& increased in Class III subjects, which was statistically significant. This was in accordance with the study of Kem et al, Angle \& Kirjavainen et al who showed decrease in Upper pharyngeal width in Class II Subjects.14 As the space between Cervical vertebrae \& mandible decreases it causes obstruction in the upper airway thus reducing the width of upper pharyngeal space anteroposteriorly. Mergen \& Jacobs reported that nasopharyngeal depth was significantly larger in patient with normal occlusion than in Class II malocclusion.4 Thus it can be concluded that as the mandible advances into more anterior position, the upper pharyngeal width also increases. Thus with the increase in ANB there is subsequent decrease in upper pharyngeal width \& Vice-versa.

Adenoids also referred to as nasopharyngeal tonsils, is a part of Waldevers ring which is situated in the nasopharvngeal area. 
Adenoids are classified under lymphoid tissues, which show the growth regression after particular period of time according to Scammons growth curve. ${ }^{14}$ Upper aerial width \& lower aerial width determines the dimensions of adenoidal space, with no significant correlation to any changes in ANB. This might be the cause of fact that the sample that was choosen for the study were between the age of 15-25 years in which the nasopharynx has already achieved the adult size $\&$ by time the adenoid tissue growth is also regressed to achieve the average upper pharyngeal space dimensions.

Lower pharyngeal airway was found to be increased in Class III malocclusion. It was found to be normal in Class II malocclusion as the functional adaptation to reduced upper airway otherwise the posterior oropharyngeal and laryngeal spaces might get encroached. This was in accordance with the study conducted by Tourne in 1991 who showed increase in lower airway space in Class III subjects. ${ }^{15}$

The vertical position of hyoid bone in relation to mandibular plane was found upward in Class I subjects and Class III subjects and downwards in class II malocclusion As the upper airway is compromised in Class II subjects the downward position of hyoid bone is the anatomic adaptation to maintain the stability and patency of the pharyngeal airway. The anteroposterior distance between nasion and hyoid bone when measured on Frankfort horizontal plane, was found to be increased in Class II subjects and reduced in Class III subjects, signifying anteriorly positioned hyoid bone in Class III subjects and posteriorly postioned hyoid bone in Class II subjects.

NSH angle was found to be increased in Class II malocclusion and decreased in Class III malocclusion which signifies posterioly positioned hyoid bone in Class II malocclusion and anterior positioning of hyoid bone in Class III malocclusion. Both the finding were in accordance with the study done by Ceylan and Oktay 1995 who showed that with an increase in ANB angle, the distance between fourth cervical vertebra and hyoid bone decreased i.e posteriorly positioned hyoid bone in Class II malocclusion.16 According to the study done by Kurdo et al, 1966 the body of hyoid bone was located backward in Class II samples and forward in Class III samples in comparison to control group.17 Adamidis and Spyropoulos,1992 reported a more anterior position and decreased inclination of the hyoid bone in Class III group thus supporting the results of the present study. ${ }^{18}$ Significant increase in thickness of soft palate was seen in Class III group \& reduced in Class II group. The backward position of the tongue in subjects with retrognathic mandible pushed the soft palate posterior \& decreased the dimension of upper airway. Reduced thickness of soft palate is because of the forces exerted by the tongue over the soft palate. Reduction in the thickness of soft palate will further maintain the patent airway in upper pharyngeal space which is otherwise reduced in Class II group as per statistics. This was in accordance with the study conduct by Elham \& Susan and Jena AK, 2010 in which they showed the decrease in the thickness of soft palate with the increase in $\mathrm{ANB}^{5}$.
No significant changes were seen in the inclination of soft palate with the changes in ANB. This could be due to the functional adaptation of the tongue position along with the change in position of mandible associated with various skeletal malocclusions, thus nullifying the anteposterior skeletal pattern.

\section{Conclusion:}

Upper pharyngeal airway width was reduces with the increase in ANB. Lower pharyngeal airway width increases with the decrease in ANB. Velopharyngeal airway increases with the increase in ANB. Hyoid bone was found to be positioned posteriorly and inferiorly in with the increase in ANB. Thickness of soft palate was decreases with the increase in ANB. Therefore, the pharyngeal airway dimensions, thickness of soft palate and position of hyoid bone have a significant correlation with the changes in ANB in Uttrakhand population. Futher study is suggested to assess whether new norms for pharyngeal airway and fot the position and size of adjacent structures should be established for this very population to consider the values during diagnosis and treatment planning.

\section{References:}

1. Soheilifer S. Soheilifer S. Soheilifer S. Upper airway dimensions in patients with Class I and Class II skeletal pattern. Avicenna J Dent Res.December 2014; 6(2);e23300.

2. Maghsoudi S. Azerbayejani S. Cephalometric analysis of hyoid bone position in different jaw dysplasias. J Res Dent sci.2015; 11(4):220-228.

3. Gray H. Anatomy of human body.edi 40. September 2007; pg 194-195.

4. Amayeri M. Saleh F. Saleh M. The position of hyoid bone in different facial patterns: A lateral cephalometric study. J Uni Beirut. 2013.

5. Allhaija ESA. Al-Khateeb SN. Uvulo-glosso-pharyngeal dimentions in different anteroposterior skeletal patterns. Angle Orthodontist. 2005; vol 75:no 6.

6. Indriksone I. Jakobsone G. The upper airway dimensions in different sagittal cariofacial patterns: A systemic review. Baltic dental and maxillofacial journal. 2014; 16:109-17.

7. Calleja GZ. Human adaptation to high altitude and to sea level. Edi 1. Chap C. July 2007; pg 25-27.

8. Samman N. Mohammadi H. Xia J.Cephalometric norms for upper airway in a healthy Hong Chinese population. Hong Kong Med J. 2003; 0:25-30.

9. Oktay H. A comparison of ANB, WITS, AF-BF, and APDI measurements. Am J Orthod Dentofacial Orthop. 1991; 99:122128.

10. Ishikawa H. Nakamura S. Iwasaki H. Kitazawa S. Seven parameters describing anteroposterior jaw relationship; 
postpubertal prediction accuracy and interchangeability. Am J Orthod Dentofacial Orthop. 2000; 117:714-720.

11. Jacobson A. The Wits appraisal of jaw disharmony. Am J Orthod.1975; 67:125-138.

12. Hussel W. Nanda RS. Analysis of factors affecting angle ANB. Am J Orthod.1984; 85:411-423.

13. Solow B. Siersbaek-Neilsen S. Greve E. Airway adequacy, head posture, and craniofacial morphology. Am J Orthod.1984; $86: 214-223$.

14. Yadav D. Rai D.Gill V. Dosi A. Choudhary A. Importance and calculation of pharyngeal space and hyoid position before and after Orthognathic surgery. AHB.2015; 5(1):5-13.
15. Tourne LPM. Growth of the pharynx and its physiologic implications. Am J Orthod Dentofacial Orthop. 1991; 99:129. 139.

16. Ceylan I, Oktay H. A study on the pharyngeal size in different skeletal patterns. Am J Orthod Dentofacial Orthop. 1995; 108(1):69-75.

17. Kurdo T. Nunota E. Hanada K. Ito G. Shibasaki Y. A roentgenocephalometric study on the position of hyoid bone. Bull Tokyo Med Dent Uni.1966; 13:227-43.

18. Adamidis IP. Spyropoulos MN. Hyoid bone position in response to mandibular protrusion in Class I and Class III malocclusions. Am J Orthod Dentofacial Orthop. 1992; 101:308312.

Cite This Article As: Nazir U, Basu R, Pranshu, Agrawal

R. Assessment of Pharyngeal Width and Length, Position of Hyoid Bone, It's size and Inclination of Soft Palate in Different Skeletal Malocclusions in Uttarakhand Population SJCMDS 2021; 1(1):1-13. 